Reprod. Nutr. Dévelop. 1980, 20 (2), 587-591.

\title{
ABP : la protéine testiculaire de liaison des androgènes.
}

par M. COUROT

Laboratoire de Fertilité mâle, I.N.R.A.

Nouzilly 37380 Monnaie France.

\section{Summary. Androgen binding protein.}

ABP is a protein found in the testicular cytosol or secreted by Sertoli cells in the rete testis fluid. It has a high affinity for androgers and binds specifically $5 \alpha-D H T$ and testosterone (but to a lesser extent). The binding capacity is saturable. ABP is measured by steady state polyacrylamide gel electrophoresis or by separation on dextran coated charcoal.

$A B P$ moves from testis to epididymis where its binding activity is totally or partially destroyed from caput to cauda epididymis. In some species (ram, buil, billy goat) but not in others (human, boar, stallion) ABP is present in the seminal plasma of the ejaculate.

In some species like the rat, ABP is also secreted in the testicular blood stream by Sertoli cells through the basement membrane of the seminiferous tubules.

$A B P$ varies with age and with season. Its production is under separate endocrine control of FSH and testosterone and its transport from testis to epididymis is specifically controlled by FSH.

Through its binding activity, ABP may play a role in spermatogenesis and epididymal sperm maturation by enhancing the local concentration of androgens around the germinal cells and the male gametes. However ABP is not present in some species, like the pig, although their spermatogenesis and epididymal sperm maturation are normal.

L'ABP (« Androgen Binding Protein ») est une glycoprotéine de liaison des androgènes qui a été mise en évidence dans le cytosol testiculaire ou épididymaire dans un certain nombre d'espèces par électrophorèse sur gel de polyacrylamide. Elle présente une affinité élevée pour la $5 \alpha$ dihydrotestostérone $(5 \alpha \mathrm{DHT})\left(\mathrm{Ka}=1,25.10^{9} \mathrm{M}^{-1}\right)$ et la testostérone $\left(\mathrm{Ka}=0,5.10^{9} \mathrm{M}^{-1}\right)$ (Hansson et al., 1974). Elle lie spécifiquement ces deux stéroïdes androgènes, principalement la $5 x$ DHT et en moindre quantité la testostérone, mais très peu ou pas du tout les autres stéroïdes, qu'ils soient d'origine gonadique ou surrénalienne (Hansson ef al., 1975 ; Jegou et al., 1978). Sa capacité de liaison est limitée. C'est une protéine thermolabile, sensible à la trypsine. D'un poids moléculaire de 70 à 90000 daltons, elle se compose de 2 sous-unités de 40000 environ (Musto, Gunsalus et Bardin, 1978). On admet qu'elle comporte un site de liaison par molécule d'ABP.

Purification et dosage : Jusqu'à présent peu d'équipes ont réussi à obtenir de l'ABP assez purifiée pour préparer un anticorps (Weddington ef al., 1975 ; Gunsalus, Musto ef Bardin, 1978). L'une d'elles a pu développer un dosage radioimmunologique de 
I'ABP de rat (Gunsalus, Musto et Bardin, 1978). Pour tous les autres travaux évoqués dans cette courte revue, les auteurs ont dosé l'ABP par la méthode au charbon dextran ou par électrophorèse à l'équilibre en gel de polyacrylamide (Ritzen et al., 1971).

Origine : l'ABP est une protéine d'origine testiculaire : on l'observe en effet dans le cytosol du testicule (Hansson ef al., 1973a), elle est excréfée dans le fluide testiculaire (Jegou ef al., 1978) et passe dans l'épididyme d'où elle disparaît si on ligature les canaux efférents (French et Ritzen, 1973). Plus précisément, l'ABP est produite par les cellules de Sertoli (Hagenäs et al., 1978 ; Steinberger et Steinberger, 1977 ; Rommerts ef al., 1977) dont on peut considérer qu'elle est un marqueur spécifique. La production d'ABP est très altérée dans le testicule cryptorchide (Hagenäs et al., 1978).

Devenir : La voie de sécrétion principale de l'ABP est le fluide testiculaire qui transporte la protéine vers l'épididyme. Sa concentration exprimée par sa capacité à lier la $5 \propto$ DHT à saturation (pmoles $/ \mathrm{mg}$ de protéines dans le surnageant $105000 \mathrm{~g}$ ) diminue progressivement de la tête ò la queue de l'épididyme chez le rat adulte (Hansson et al., 1974). Ceci traduit une destruction de la capacité de liaison de la protéine au cours de son passage dans l'épididyme. Cette observation est confirmée chez le lapin adulte par la comparaison des concentrations (molaires) d'ABP dans le fluide testiculaire ef celui de la queue épididymaire (Danzo, Cooper et Orgebin-Crist, 1977). Chez le bélier aussi, on montre une destruction de la protéine dans l'épididyme mais on doit, pour cela, prendre en compte les débits des fluides et calculer la quantité d'ABP circulant à travers l'épididyme car sa concentration est plus élevée dans le fluide de la queuede l'épididyme que dans le fluide testiculaire (Jegou et al., 1979). La présence d'ABP dans le plasma séminal de l'éjaculat a été observée chez certaines espèces (taureau, bélier, bouc) alors qu'on ne peut pas la détecter chez d'autres (homme, verrat, étalon) (Jegou ef Le Gac-Jegou, 1978).

Chez certaines espèces, l'ABP est aussi sécrétée par les cellules de Sertoli à travers la membrane basale des tubes séminifères dans le circuit sanguin du testicule. Dans les espèces comme le rat qui n'ont pas de globuline de liaison de la testostérone et de l'œstradiol, on a pu mesurer la quantité d'ABP présente dans le sang périphérique (Gunsalus, Musto et Bardin, 1978). En dépit de sa faible concentration plasmatique la quantité de cette protéine dans le volume sanguin total est équivalente à 45 p. 100 du contenu testiculaire et 15 p. 100 du contenu épididymaire chez le rat. Si dans cette espèce, elle disparaît après castration avec une demie-vie de 1 jour (Gunsalus, Musio et Bardin, 1978), chez le bélier, la castration n'entraîne aucune variation de la sécrétion en protéines de liaison des androgènes présentes dans le sang (Jegou, communication personnelle).

\section{Facteurs de variation.}

- Effef de l'âge. L'ABP a pu être détectée dans le testicule de l'agneau infantile bien avant la différenciation morphologique des cellules de soutien en cellules de Sertoli. La protéine est observée aussi au cours de l'établissement de la spermatogenèse et dans le testicule adulte. Sa concentration par mg de protéine diminue avec le développement de la gonade en raison de la «dilution » par les cellules germinales, des cellules de Sertoli qui la produise ; mais, lorsque cette concentration est rapportée au nombre de cellules de Sertoli, on constate qu'elle augmente jusqu'à l'âge adulte chez le 
bélier (Carreau, Drosdowsky et Courot, 1979). Chez le rat, contrairement à l'agneau, la concentration d'ABP par $\mathrm{mg}$ de protéines testiculaires augmente au cours de la période prépubère et diminue seulement après la puberté (Tindall, Vitale et Means, 1975) montrant ainsi qu'il existe des différences entre espèces quant à la dynamique de la production d'ABP en fonction de l'âge.

Dans l'épididyme, l'ABP apparaît lorsque s'est formée la lumière des tubes séminifères qui permet au fluide testiculaire de transporter la protéine vers l'épididyme. La concentration d'ABP par $\mathrm{mg}$ de protéines épididymaires augmente avec l'âge chez le rat et le bélier (Hansson et al., 1973b ; Tindall, Vitale et Means, 1975 ; Carreau, Drosdowsky et Courot, 1979) mais pas chez le lapin où Danzo, Cooper et Orgebin-Crist (1977) observent une diminution de l'ABP dans le fluide de la queve de l'épididyme chez l'animal adulte par rapport à l'immature.

- Effet de la saison. Dans les espèces qui présentent une variation saisonnière de l'activité sexuelle comme le bélier, la concentration d'ABP dans le testicule est significativement plus élevée en saison sexuelle qu'en contre saison (Jegou ef al., 1978). Cette différence est notée tout au long de l'appareil génital et jusque dans le plasma séminal du sperme éjaculé (Jegou ef al., 1979) suggérant que la mesure de cette protéine dans l'éjaculat peut représenter un moyen commode d'apprécier l'activité des cellules de Sertoli dont elle provient.

Contrôle endocrinien : Dès les premiers travaux sur l'ABP, il est apparu que la protéine est sous contrôle endocrinien puisqu'elle disparaît du testicule après hypophysectomie et qu'un traitement combiné de FSH et LH en restaure la production chez le rat (Hansson ef al., 1973b el 1974). On a aussi montré que LH agit par l'intermédiaire de la testostérone qu'elle fait sécréter, mais tous les auteurs ne s'accordent pas sur la dépendance hormonale de l'ABP : certains donnent un rôle prioritaire à FSH alors que pour d'autre, la testostérone est essentielle. Peut être est-ce dû à des différences entre les effets rapides et transitoires et les effets à long terme des hormones; ces derniers mettraient en cause une stimulation de la synthèse de novo de l'ABP (Louis et Fritz, 1979). Selon les travaux les plus récents réalisés sur des cellules de Sertoli de rat immałure cultivées in vifro, ces deux hormones, $\mathrm{FSH}$ et festostérone, augmentent la sécrétion de I'ABP. Elles agissent indépendamment l'une de l'autre avec, toutefois, une interaction significative lorsqu'elles sont administrées simultanément (Louis et Fritz, 1979).

Par contre, le transport de l'ABP dans l'épididyme semble spécifiquement contrôlé par FSH (Weddington et al., 1975 ; Kotite, Nayfeh et French, 1978 ; Tindall, Mena et Means, 1978). Néanmoins, une corrélation positive a été signalée entre la teneur du fluide testiculaire ou du fluide des canaux efférents en ABP et leur teneur en $5 \alpha$ DHT ou en testostérone chez le lapin ef le bélier (Guerrero ef al., 1975 ; Jegou ef al., 1979). La comparaison entre espèces montre aussi une corrélation entre les concentrations du fluide testiculaire en $A B P$ et en testostérone (Ritzen et al., 1977).

Rôle : L'ABP lie spécifiquement les androgènes, mais les stéroïdes liés se dissocient assez rapidement de la protéine porteuse $(+1 / 2=6 \mathrm{mn}$ à $0 \circ \mathrm{C})$. Ceci suggère que cette protéine doit jouer un certain rôle dans le stockage et le transport des androgènes vers les organes cibles de l'appareil génital mâle. En premier lieu, en raison de la concentration des stéroìdes qu'elle assure à proximité immédiate des cellules germinales dans l'épithélium séminifère, on a pensé que l'ABP intervient dans le fonctionnement de la 
spermatogenèse dont on connaît l'androgéno-dépendance chez le rat (Steinberger, 1971). En second lieu, une partie de l'ABP est transportée par le fluide testiculaire vers l'épididyme où elle pourrait avoir un rôle physiologique dans la maturation des spermatozoïdes en contribuant à augmenter la concentration d'androgènes dans l'épididyme. La maturation épididymaire est en effet connue comme étant elle aussi sous la dépendance des androgènes (Orgebin-Crist, Danzo et Davies, 1975).

On doit cependant remarquer que, pour aussi plausible qu'il puisse paraître, le rôle physiologique de l'ABP chez le mâle reste spéculatif car aucune preuve expérimentale rigoureuse n'en a encore été apportée... D'autre part, il ne semble pas que la présence d'ABP soit générale car on n'en a pas trouvé dans certaines espèces comme le verrat chez qui, cependant, l'activité spermatogénétique ef la maturation épididymaire sont tout à fait normales.

Présenté au Colloque D.G.R.S.T. de Porf Bail, 27 février-1 er mars 1979.

Accepté en ao0t 1979.

\section{Références}

CARREAU S., DROSDOWSKY M. A., COUROT M., 1979. Age related effects on androgen binding protein (ABP) in sheep testis and epididymis. Int J. Androl., 2, 49-61.

DANZO B. J., COOPER T. G., ORGEBIN-CRIST M. C., 1977. Androgen binding protein (ABP) in fluids collected from the rete testis and cauda epididymis of sexually mature and immature rabbits and observations on morphological changes in the epididymis following ligation of the ductuli efferentes; Biol. Reprod., 17, 64-77.

FRENCH F. S., RITZEN E. M., 1973. A high affinity androgen-binding protein (ABP) in rat testis : evidence for secretion into efferent duct fluid and absorption by epididymis. Endocrinology, 93, 88-93.

GUERRERO R., RITZEN E. M., PURVIS K., HANSSON V., FRENCH F. S., 1975. Concentration of steroid hormones and androgen binding protein (ABP) in rabbit efferent duct fluid., 213221. In FRENCH F. S., HANSSON V., RITZEN E. M., NAYFEH S. N. Hormonal regulation of spermatogenesis, Plenum Press.

GUNSALUS G. L., MUSTO N. A., BARDIN C. W., 1978. Immunoassay of androgen binding profein in blood : a new approach for study of the seminiferous fubule. Science, 200, 65-66.

HAGENÄS L., RITZEN E. M., SVENSSON J., HANSSON V., PURVIS K., 1978. Temperature dependance of Sertoli cell function. Int. J. Androl. Suppl., 2, 449-458.

HANSSON V., DJOSELAND O., REUSCH E., ATTRAMADAL A., TORGERSEN O., 1973a. An androgen binding protein in the testis cytosol fraction of adult rats. Comparison with the androgen binding protein in the epididymis. Steroids, 21, 457-474.

HANSSON V., REUSCH E., TRYGSTAD O., TORGERSEN O., RITZEN E. M., FRENCH F. S., 19736. FSH stimulation of testicular androgen binding protein. Nature New Biol., 246, 56-58.

HANSSON V., TRYGSTAD O., FRENCH F. S., M; LEAN W. S., SMITH A. A., TINDALL D. J., WEDDINGTON S. C., PETRUSZ P., NAYFEH S. N., RITZEN E. M., 1974. Androgen transport and receptor mechanisms in testis and epididymis. Nature, 250, 387-391.

HANSSON V., RITZEN E. M., FRENCH F. S., NAYFEH S. N., 1975. Androgen transport and receptor mechanisms in testis and epididymis, 173-201. In GREEP R. O., HAMILTON D. W., GEIGER S. R., Handbook of Physiology, Endocrinology, V, Am. Physiol. Soc. Publ.

JEGOU B., LE GAC-JEGOU F., 1978. Androgen-binding protein in the seminal plasma of some mammalian species. J. Endocr., 77, 267-268.

JEGOU B., DACHEUX J. L., TERQUI M., GARNIER D. H., COUROT M., 1978. Studies of the androgen binding protein in the rete testis fluid of the ram and its relation to sexual season. Mol. cell. Endocr., 9, 335-346. 
JEGOU B., DACHEUX J. L., GARNIER D. H., TERQUI M., COLAS G., COUROT M., $1979 . \quad$ Biochemical and physiological studies of androgen binding protein in the reproductive tract of the ram. J. Reprod. Fert., 57, 311-318.

KOTITE N. J., NAYFEH S. N., FRENCH F. S., 1978. FSH and androgen regulation of Sertoli cell function in the immature rat. Biol. Reprod., 18, 65-73.

LOUIS B. G., FRITZ I. B., 1979. Follicle stimulating hormone and testosterone independently increase the production of androgen binding protein by Sertoli cells in culture. Endocrinology, 104, 454-462.

MUSTO N. A., GUNSALUS G. L., BARDIN C. W., 1978. Further characterization of androgen binding protein in epididymis and blood. Int. J. Androl., Suppl. 2, 424-433.

ORGEBIN-CRIST M. C., DANZO B. J., DAVIES J., 1975. Endocrine control of the development and maintenance of sperm fertilizing ability in the epididymis, 319-338, in GREEP R. O., HAMILTON D. W., GEIGER S. R., Handbook of Physiology, Endocrinology, V, Am. Physiol. Soc. Publ.

RITZEN M. E., NAYFEH F. S., FRENCH F. S., DOBBIN M. C., 1971. Demonstration of androgen binding components in rat epididymis cytosols and comparison with binding components in rat prostate and other tissues. Endocrinology, 89, 143-151.

RITZEN E. M., HAGENÄS L., PURVIS K., GUERRERO R., JOHNSONBAUGH R. E., DYMM., FRENCH F. S., HANSSON V., 1977. Androgens and androgen binding protein (ABP) in testicular fluids, 79.87, in BIERSCH J. R., RAGER K., RANKE M. B. Maldescendus testis, Urban et Schwarzenberger Publ. Munich.

ROMMERTS F. F. G., KRÜGER-SEWNARAIN B. Ch., GROOTEGOED J. A., Van der MOLEN H. J., 1977. Analysis of androgen-binding protein in media from Sertoli cell incubations and cytosols from rat testis. J. Endocr., 73, 511-517.

STEINBERGER E., 1971. Hormonal control of mammalian spermatogenesis. Physiol. Rev., 51, 1-12.

STEINBERGER A., STEINBERGER E., 1977. The Sertoli cells, 371-399, in JOHNSON A. D., GOMES W. R., The Testis, vol. 4, Acad. Press.

TINDALL D. J., MENA C. R., MEANS A. R., 1978. Hormonal regulation of androgen binding protein in hypophysectomized rats. Endocrinology, 103, 589-594.

TINDALL D. J., VITALE R., MEANS A. R., 1975. Androgen binding protein as a biochemical marker of formation of the blood-testis barrier. Endocrinology, 97, 636-648.

WEDDINGTON S. C., BRANDTZAEG P., HANSSON V., FRENCH F. S., PETRUSZ P., NAYFEH S. N., RITZEN E. M., 1975. Immunological cross reactivity between testicular androgen-binding protein and serum testosterone-binding globulin. Nature, 258, 257-259. 